Original Article - Clinical Science

\title{
Prevalence, associations and characteristics of severe uncorrected refractive error in the Australian National Eye Health Survey
}

\author{
Stuart Keel PhD, ${ }^{1}$ Myra B. McGuiness PhD, ${ }^{1}$ J oshua Foreman PhD, ${ }^{1,2}$ J ane Scheetz \\ $\mathrm{PhD},{ }^{1}$ Hugh R. Taylor FRANZCO AC ${ }^{3}$ and Mohamed Dirani PhD ${ }^{1,4}$
}

${ }^{1}$ Centre for Eye Research Australia, Royal Victorian Eye \& Ear Hospital, Melbourne, Australia

2Ophthalmology, Department of Surgery, University of Melbourne, Melbourne, Australia

3Indigenous Eye Health Unit, Melbourne School of Population and Global Health, The University of Melbourne, Melbourne, Australia

${ }^{4}$ Singapore Eye Research Institute, Singapore National Eye Centre, Singapore.

Correspondence: Dr Stuart Keel, Level seven, Centre for Eye Research Australia, 32 Gisborne Street, East Melbourne, Victoria, Australia 3002

Email: $\quad$ stuart.keel@unimelb.edu.au

Short running title: Prevalence of uncorrected refractive error Received 31 J anuary 2019; accepted 21 September 2019

Funding sources / Financial disclosure: Department of Health of the Australian Government, Novartis Australia and the Peggy and Leslie Cranbourne Foundation. In-kind support from our industry and sector partners, OPSM, Carl Zeiss, Designs for This is the author manuscript accepted for publication and has undergone full peer review but has not been through the copyediting, typesetting, pagination and proofreading process, which may lead to differences between this version and the Version of Record. Please cite this article as doi: $10.1111 /$ ceo.13647

This article is protected by copyright. All rights reserved. 
Vision, the Royal Flying Doctor Service, Optometry Australia and the Brien Holden Vision Institute. The Centre for Eye Research Australia receives Operational Infrastructure Support from the Victorian Government. The Principal Investigator, Dr Mohamed Dirani, is supported by a NHMRC Career Development Fellowship (\#1090466). The PhD student, Joshua Foreman is supported by an Australian Postgraduate Award scholarship.

Conflict of interest: None 


\section{ABSTRACT}

Importance: In Australia, nationally representative data of the burden and associations of severe uncorrected refractive error is scarce.

Background: To report the prevalence and characteristics of severe uncorrected refractive error in Indigenous and non-Indigenous Australians.

Design: Population-based cross-sectional study

Participants: 3098 non-Indigenous Australians aged 50-98 and 1738 Indigenous Australians aged 40-92 living in 30 randomly selected Australian sites were examined.

Methods: Severe uncorrected refractive error was defined as an improvement of $\geq 2$ lines on the logMAR chart in one or both eyes in participants with a presenting visual acuity $<6 / 12$.

Main outcome measure: Severe uncorrected refractive error

Results: Prevalence of severe uncorrected refractive error was $11.0 \%$ (95\% Cl 9.313.0) in non-Indigenous and $14.5 \%$ (12.5-16.7) in Indigenous Australians. Eightytwo percent of non-Indigenous and $77 \%$ of Indigenous participants had a spherical equivalent refraction between $-2.00 \mathrm{D}$ and $+2.00 \mathrm{D}$. Indigenous Australians who were older in age (OR for 70-79yrs vs. 40-49yrs $=3.59$ ), resided in outer regional areas $(\mathrm{OR}=1.78)$ and didn't have an eye examination in the previous 2 -years $(\mathrm{OR}=1.50)$ were associated with higher odds of severe uncorrected refractive error. Geographical remoteness ( $O R=0.68$ for inner regional), male gender $(O R=1.30$ ), older age (OR for $70-79 y$ rs vs. $50-59 y r s=1.51$ ) and failure to have an eye examination in the previous 2-years $(O R=2.06)$ were associated with severe uncorrected refractive error among non-Indigenous participants.

Conclusions and relevance: Increased public awareness of the importance of regular optometric examinations may be required in groups at high risk of severe uncorrected refractive error. 
Key words: uncorrected refractive error, epidemiology, vision loss

\section{I NTRODUCTI ON}

Population-based surveys have consistently reported high rates of uncorrected refractive errors amongst adult populations in Australia ${ }^{1,2}$ and internationally. ${ }^{3-7}$ It has been long established that uncorrected refractive error significantly impacts quality of life (QoL) and independent living. ${ }^{8,9}$ Given the feasibility of treatment in most cases with new or updated spectacle correction, knowledge of the burden and associations of severe uncorrected refractive error is useful to inform targeted resource allocation.

In Australia, two population-based studies conducted in the early 1990s, the Melbourne Visual Impairment Project (VIP) that surveyed Australians aged $\geq 40$ years ${ }^{10}$ and the Blue Mountains Eye Study (BMES) that surveyed Australians aged $\geq 49$ years, ${ }^{11}$ documented the prevalence of uncorrected refractive error to be $10.2 \%$ and $10.0 \%$, respectively. Despite this, robust comparisons between these studies are problematic due to differing definitions of uncorrected refractive error and age inclusion criterions employed. The National Indigenous Eye Health Survey (NIEHS, 2008) and the Central Australian Ocular Health Study (CAOHS, 2008) reported that uncorrected refractive error is the main cause of over half of all visual impairment cases in Indigenous adults aged $\geq 40$ years. ${ }^{12,13}$

Decades on, our recent report from Australia's National Eye Health Survey (NEHS) found that refractive error remains the leading cause of visual impairment among Indigenous and non-Indigenous Australian adults, accounting for $63.5 \%$ and $61.7 \%$ of cases, respectively. ${ }^{14}$ In the present study, we aim to undertake further analysis 
to elucidate prevalence and factors associated with severe uncorrected refractive error ( $\geq 2$ lines improvement in subjects with a presenting vision $<6 / 12$ ). Furthermore, we aim to report the distribution of spherical equivalent and cylindrical refractive errors in this population. It is hoped that these data may be useful for informing interventions targeting the more severe cases of uncorrected refractive error in Australia.

\section{METHODS}

\subsection{Study population}

The National Eye Health Survey was a population-based survey (March 2015 - April 2016) of non-Indigenous Australians, aged 50 years and older, and Indigenous Australians, aged 40 years and older. The younger age criteria for Indigenous participants was chosen due to the earlier onset and more rapid progression of common eye diseases and diabetes in Indigenous Australians. ${ }^{15}$ The detailed sampling and testing methodology has been reported elsewhere. ${ }^{16,17}$ Multistage random-cluster sampling was used to select 30 population clusters of 150 participants (100 non-Indigenous and 50 Indigenous per sampling site). Selection of sites utilised Census 2011 data collected by the Australian Bureau of Statistics (ABS) that categorised Statistical Areas into five Remoteness Areas (RAs); Major City, Inner Regional, Outer Regional, Remote and Very Remote. Door-to-door recruitment was conducted until the target sample size was reached in each cluster. In total, 11,883 residents were contacted across 30 population clusters. Of these, 6,760 (56.9\%) were eligible to participate in the survey and a total of 5,764 agreed to participate, resulting in a positive response rate of $85.3 \%(5,764 / 6,760)$. Of these, 4,836 residents attended NEHS testing venues and underwent examinations, resulting in an overall clinical examination rate of $71.5 \%(4,836 / 6,760)$. Ethics approval was obtained from the Royal Victorian Eye and Ear Hospital (RVEEH) Human Research 
Ethics Committee (HREC-14/1199H) and additional ethical approvals were obtained at the State level to conduct research within Indigenous communities. Study procedures adhered to the tenets of the Declaration of Helsinki as revised in 2013 and participants provided written informed consent to participate.

\subsection{Examination procedures}

Socio-demographic data, utilisation of eye health services, as well as medical and ocular histories were collected via an interviewer-administered questionnaire. Selfreported use of near, distance or bifocal corrective lenses were also recorded at the time of examination. All tests were conducted by orthoptists, optometrists, ophthalmologists or research assistants who were thoroughly trained using a standardized training protocol. Presenting distance visual acuity (PVA) was measured first in the right eye and then the left eye using a logMAR chart (Brien Holden Vision Institute) in well-lit room conditions. Participants wore their usual distance correction if available at the time of examination. Starting at the $6 / 60$ line, participants were asked to read the first letter on the line and continue reading down the chart until difficulty was observed. A line was achieved if $\geq 3$ letters were correctly identified. Light perception was assessed using a pen torch for participants who could not discern the 6/60 letters. For all participants with PVA $<6 / 12$ in one or both eyes, pinhole testing was performed and, if visual acuity (VA) improved in either eye, an auto-refraction was then conducted using a Nidek ARK-30 Type-R hand-held autorefractor (Nidek Co, Japan). Visual acuity was re-measured with the auto-refractor correction placed in the trial lens frame. Best corrected VA was defined as the best of the pinhole or automated refraction measurements for each eye.

Examination of the anterior segment of all eyes was performed using a hand-held slit lamp (Keeler Ophthalmic Instruments, UK). Participants with PVA $<6 / 12$ in one or both eyes had anterior segment photographs taken of the eye(s) with reduced visual acuity using a Digital Retinography System (DRS) camera (CenterVue SpA, 
Italy) to be used for subsequent attribution of the likely cause of vision loss. For all participants, two, non-stereoscopic, 45o colour fundus photographs were taken of each retina, centred on the macula and optic disc, respectively, using the DRS nonmydriatic camera. Pupillary dilatation was conducted when retinal images were of reduced quality due to small pupil size. The diagnosis of cataract was made by the study optometrists based on protocols described in detail elsewhere. ${ }^{18}$ Trained retinal graders graded images for diabetic retinopathy (DR) as described in detail elsewhere ${ }^{19}$ and age-related macular degeneration (AMD) ${ }^{20}$ according to standard protocols. A diagnosis of glaucoma was ascertained by fellowship-trained glaucoma specialists as described in detail elsewhere. ${ }^{21}$

\subsection{Definition of severe uncorrected refractive error}

Participants were considered to have severe uncorrected refractive error if the distance best-corrected VA improved by $\geq 2$ lines on the logMar chart in one or both eyes in subjects with a PVA of $<6 / 12$. This included participants with suboptimal correction of their refractive error. The number of lines of improvement was calculated as the best-corrected VA minus the PVA. The spherical equivalent (SE) of auto-refraction results, which is the sum of the sphere plus half of the cylindrical value, was calculated to provide one numerical value for the refractive error of each participant.

\subsection{Statistical analysis}

Population proportions were estimated using post-stratification for age in addition to the survey weights which were derived separately for Indigenous and nonIndigenous participants according to study site. Population values were derived using data from the 2016 Australian Census (source: Australian Bureau of Statistics TableBuilder <www.abs.gov.au>, accessed 19 April 2018). 
For each participant, the eye with the worst presenting visual acuity was selected for all analysis. Age and sex adjusted logistic regression was performed separately for Indigenous and non-Indigenous participants to investigate the following risk factors for severe uncorrected or sub-optimally corrected refractive error (per person): educational attainment (less than high school, completed high school, completed trade or university qualification), main language spoken at home (English, language other than English), ethnicity (for non-Indigenous participants only; Oceanian, European, Other), remoteness area (major city, inner regional, outer regional, remote, very remote), self-reported diabetes (yes versus no) and time since last eye exam. Contrasts of the odds of severe refractive error were assessed for trends across categories of age in ten-year groupings. Age and sex adjusted logistic regression was performed to assess the association between the presence of severe uncorrected refractive error and the following ocular conditions (per eye): cataract, history of cataract surgery, glaucoma, DR and AMD.

Cylinder power (in dioptres) was compared between Indigenous and non-Indigenous participants using the Mann-Whitney rank-sum test. All analyses were performed using Stata/SE version 15.1 (StataCorp LP, College Station, TX, USA).

\section{RESULTS}

A total of 4,836 participants were included in this analysis including 3,098 nonIndigenous (54\% female, median age 66 [interquartile range (IQR) 60, 74] years) and 1,738 Indigenous ( $59 \%$ female, median age 54, [IQR 48, 62] years) Australians. Of these, 661 (21.3\%, 453 (68.5\%) unilateral) non-Indigenous participants and 402 (23.1\%, 214 (51.1\%) unilateral) Indigenous participants had a PVA of $<6 / 12$ in one or both eyes. A total of 605/1063 had a distance best corrected VA that improved by $\geq 2$ lines and 585 (96.7\%) had available auto refraction data for analysis. 


\subsection{Weighted prevalence of severe uncorrected refractive error by Indigenous status}

The weighted prevalence of severe uncorrected refractive error in one or both eyes among non-Indigenous Australians aged 50 years and over and Indigenous Australians aged 40 years and over was $11.0 \%(95 \% \mathrm{Cl}$ 9.3,13.0) and $14.5 \%$ $(12.5,16.7)$, respectively (Table 1$)$. Bilateral severe uncorrected refractive error was present in 78 (prevalence 2.5\%) non-Indigenous participants and 91 (prevalence $5.2 \%)$ Indigenous participants. Overall, $66 \%$ (226/344) of non-Indigenous participants and 64\% (168/261) of Indigenous participants with severe uncorrected refractive error reported they didn't own glasses or contact lenses for distance. The equivalent figures for bilateral severe refractive error (only) were 68\% (53/78) and $59 \%(54 / 91)$ of non-Indigenous and Indigenous participants, respectively. 
Table 1: Estimated prevalence of severe uncorrected refractive error in one or both eyes in the National Eye Health Survey for 3098 non-Indigenous and 1738 Indigenous participants (2015-2016)

\begin{tabular}{|c|c|c|c|c|c|c|c|c|c|c|}
\hline & \multicolumn{10}{|c|}{ Prevalence of severe uncorrected refractive error } \\
\hline & \multicolumn{5}{|c|}{ Non-I ndigenous } & \multicolumn{5}{|c|}{ Indigenous } \\
\hline & \multirow{2}{*}{$\begin{array}{c}\text { Cases } \\
*\end{array}$} & \multicolumn{2}{|c|}{$\begin{array}{c}\text { Crude } \\
\text { prevalence }\end{array}$} & \multicolumn{2}{|c|}{$\begin{array}{l}\text { Weighted } \\
\text { prevalence }\end{array}$} & \multirow{2}{*}{$\begin{array}{c}\text { Cases } \\
*\end{array}$} & \multicolumn{2}{|c|}{$\begin{array}{c}\text { Crude } \\
\text { prevalence }\end{array}$} & \multicolumn{2}{|c|}{$\begin{array}{l}\text { Weighted } \\
\text { prevalence }\end{array}$} \\
\hline & & $\%$ & $\begin{array}{l}(95 \% \\
\mathrm{Cl})\end{array}$ & $\%$ & $\begin{array}{c}(95 \% \\
\mathrm{Cl})\end{array}$ & & $\%$ & $\begin{array}{l}(95 \% \\
\mathrm{Cl})\end{array}$ & $\%$ & $\begin{array}{l}(95 \% \\
\mathrm{Cl})\end{array}$ \\
\hline Total & $\begin{array}{c}344 / 30 \\
98\end{array}$ & 11.1 & $\begin{array}{l}(10.0 \\
12.3)\end{array}$ & 11.0 & $\begin{array}{l}(9.3 \\
13.0)\end{array}$ & $\begin{array}{c}261 / 17 \\
38\end{array}$ & 15.0 & $\begin{array}{l}(13.4, \\
16.8)\end{array}$ & 14.5 & $\begin{array}{l}(12.5, \\
16.7)\end{array}$ \\
\hline Sex & & & & & & & & & & \\
\hline Female & $\begin{array}{c}164 / 16 \\
61\end{array}$ & 9.9 & $\begin{array}{l}(8.5, \\
11.4)\end{array}$ & 9.7 & $\begin{array}{l}(8.0 \\
11.7)\end{array}$ & $\begin{array}{c}155 / 10 \\
24\end{array}$ & 15.1 & $\begin{array}{l}(13.1, \\
17.5)\end{array}$ & 14.3 & $\begin{array}{l}(12.1 \\
16.9)\end{array}$ \\
\hline Male & $\begin{array}{c}180 / 14 \\
37\end{array}$ & 12.5 & $\begin{array}{c}(10.9 \\
14.3)\end{array}$ & 12.6 & $\begin{array}{l}(10.2 \\
15.4)\end{array}$ & $\begin{array}{c}106 / 71 \\
4\end{array}$ & 14.8 & $\begin{array}{c}(12.4 \\
17.6)\end{array}$ & 14.7 & $\begin{array}{l}(11.9, \\
18.0)\end{array}$ \\
\hline Age (years) & & & & & & & & & & \\
\hline $40-49$ & $71 / 813$ & 8.7 & $\begin{array}{l}\text { (7.0, } \\
10.9)\end{array}$ & 9.3 & $(7.1,12)$ & $56 / 581$ & 9.6 & $\begin{array}{l}(7.5, \\
12.3)\end{array}$ & 10.2 & $\begin{array}{l}(6.8, \\
15.1)\end{array}$ \\
\hline $50-59$ & $\begin{array}{c}133 / 11 \\
68\end{array}$ & 11.4 & $\begin{array}{l}(9.7 \\
13.3)\end{array}$ & 11.6 & $\begin{array}{l}(9.0 \\
14.9)\end{array}$ & $99 / 635$ & 15.6 & $\begin{array}{l}(13.0 \\
18.6)\end{array}$ & 15.2 & $\begin{array}{l}(11.7 \\
19.4)\end{array}$ \\
\hline $60-69$ & $97 / 764$ & 12.7 & $\begin{array}{l}(10.5, \\
15.3)\end{array}$ & 12.9 & $\begin{array}{l}(10.1 \\
16.2)\end{array}$ & $65 / 363$ & 17.9 & $\begin{array}{l}(14.3, \\
22.2)\end{array}$ & 18.3 & $\begin{array}{l}(14.0, \\
23.4)\end{array}$ \\
\hline
\end{tabular}




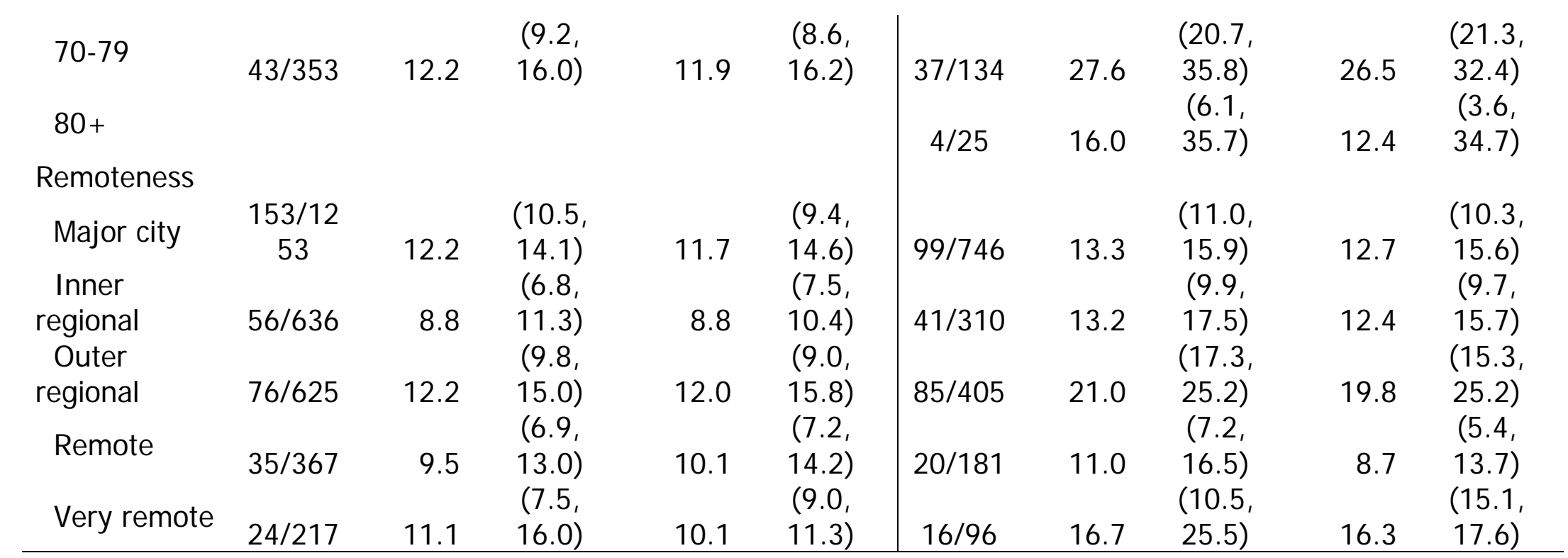

*Cases $=$ number of people with refractive error / total in category.

Weighted prevalence estimated via post-stratification for age in addition to weighting according to study site. 


\subsection{Lines improvement, spherical equivalent and cylinder auto refraction}

Following correction with pinhole or auto-refractor script (best result), VA improved to a median of $6 / 9$ [IQR; 6/7.5, 6/12] in both non-Indigenous and Indigenous participants. This corresponded to a median improvement of 3 lines [2.5,4.0] for non-indigenous participants and 3.5 lines [3.0,5.0] for Indigenous participants (Figure 1).

The spherical equivalent refractive error ranged from -9.25 to +5.25 for eyes of nonindigenous participants and -6.13 to +4.13 for eyes of Indigenous participants with severe refractive error (Table 2). Eighty-two percent of non-Indigenous and $77 \%$ of Indigenous participants with severe uncorrected refractive error had a spherical equivalent auto refraction between -2.00D and +2.00D. Among participants with severe uncorrected refractive error, there was evidence of a weak negative correlation between spherical equivalent and age for non-indigenous participants (Spearman's $\rho=-0.14,95 \% \mathrm{Cl}-0.23$ to -0.05 ) and of a weak positive correlation with age for Indigenous participants (Spearman's $\rho=0.18,95 \%$ CI 0.08 to 0.27 ; Figure 2).

There was no evidence of a difference between eyes of non-Indigenous and Indigenous participants in terms of spherical equivalent (mean difference $-0.2 \mathrm{D}$, $95 \% \mathrm{Cl}-0.5$ to 0.0 ) or cylindrical power (mean difference $-0.1 \mathrm{D}, 95 \% \mathrm{Cl}-0.2$ to 0.0 ) after adjusting for age, sex, and remoteness. Neither was there any difference in the proportion of eyes with oblique astigmatism (as defined as between 30 and 60 degrees, or between 120 and 150 degrees; 109/454, 24\% for non-Indigenous; 80/392, 20\% for Indigenous; $\chi^{2} p=0.21$ ). 


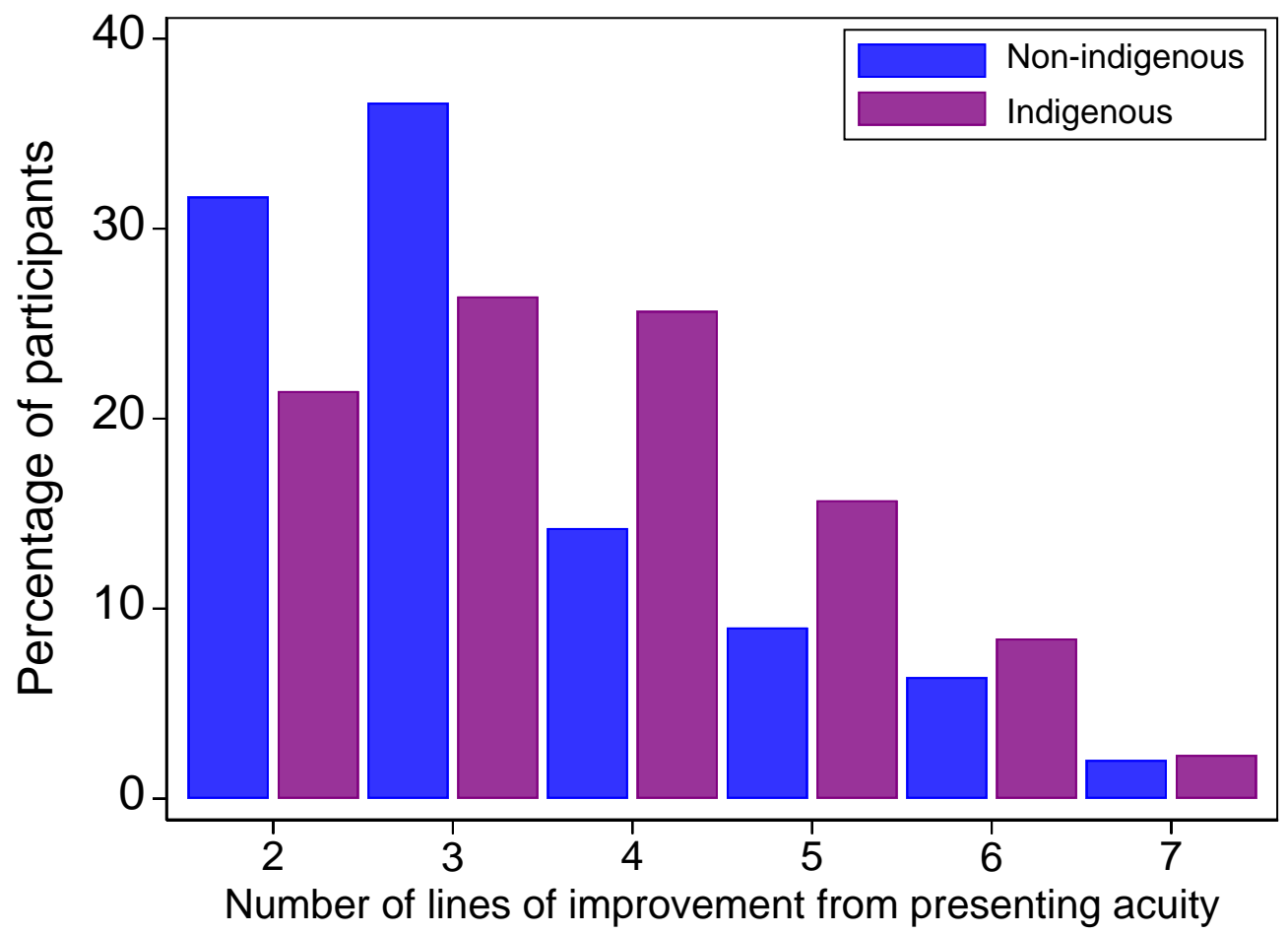

Figure 1: Distribution of number of lines improvement from presenting visual acuity for eyes with severe uncorrected refractive error.

Table 2: Distribution of spherical equivalent auto refraction in the worst eye of participants with severe uncorrected refractive error, stratified by Indigenous status

\begin{tabular}{lrcrrrrr}
\hline $\begin{array}{c}\text { Spherical } \\
\text { equivalent } \\
\text { (D) }\end{array}$ & \multicolumn{2}{c}{$\begin{array}{c}\text { Non- } \\
\text { Indigenous } \\
(\mathrm{n}=335)\end{array}$} & \multicolumn{2}{c}{\begin{tabular}{c}
\multicolumn{2}{c}{ Indigenous } \\
$(\mathrm{n}=250)$
\end{tabular}} & \multicolumn{2}{c}{$\begin{array}{c}\text { Total } \\
(\mathrm{N}=585)\end{array}$} \\
\cline { 2 - 7 } & $\mathbf{n}$ & $\mathbf{( \% )}$ & \multicolumn{1}{c}{$\mathbf{n}$} & $\mathbf{( \% )}$ & $\mathbf{n}$ & $\mathbf{( \% )}$ \\
\hline$<-2$ & 30 & $(9)$ & 34 & $(14)$ & 64 & $(11)$ \\
-2 to $<0$ & 106 & $(32)$ & 85 & $(34)$ & 191 & $(33)$ \\
0 to $<2$ & 168 & $(50)$ & 107 & $(43)$ & 275 & $(47)$ \\
$2+$ & 31 & $(9)$ & 24 & $(10)$ & 55 & $(9)$ \\
\hline
\end{tabular}




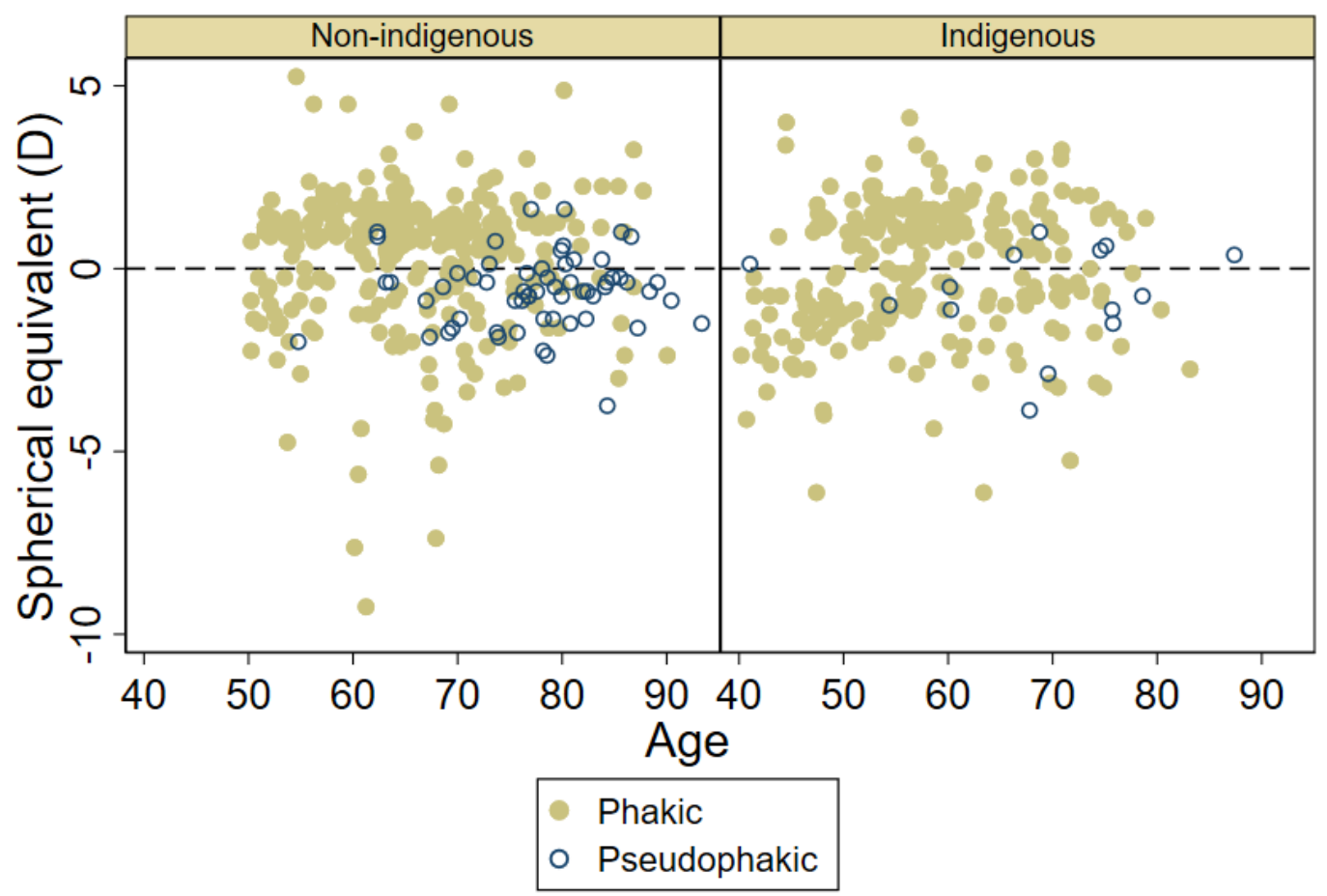

Figure 2: Relationship between spherical equivalent and age ( $n=585$ eyes from 585 participants).

\subsection{Associations of severe uncorrected refractive error}

After adjusting for age, gender and region of residence, the odds of severe refractive error were almost two times higher among Indigenous participants compared to non-Indigenous participants (adjusted OR 1.95, 95\% Cl 1.61, 2.36, p <0.001). In the non-Indigenous population, adjusted logistic regression analysis revealed that participants aged 70-79-years, males, those who had not had an eye examination in the previous 2 years or had never had an eye examination, and residing in a major city were associated with severe uncorrected refractive error. Among Indigenous participants, increasing age, residing in an outer regional remoteness area and failure to have an eye examination in the previous 2 years were associated with higher odds of severe uncorrected refractive error (Table 3). 
After adjusting for age and gender, the odds of severe uncorrected refractive error were greater among eyes with cataract for both non-indigenous and Indigenous participants. Conversely, a history of cataract surgery was associated with lower odds of severe uncorrected refractive error amongst non-Indigenous participants (Table 4). 
Table 3: Estimated association between participant characteristics and severe uncorrected refractive error in the National Eye Health Survey for 3098 non-Indigenous and 1738 Indigenous participants.

\begin{tabular}{|c|c|c|c|c|c|c|c|c|}
\hline & \multicolumn{4}{|c|}{ Non-I ndigenous } & \multicolumn{4}{|c|}{ Indigenous } \\
\hline & 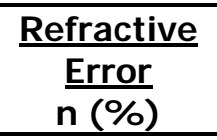 & OR & $(95 \% \mathrm{Cl})$ & $\mathbf{p}$ & $\begin{array}{c}\text { Refractive } \\
\text { Error } \\
\text { n(\%) }\end{array}$ & OR & $(95 \% \mathrm{Cl})$ & $\mathbf{P}$ \\
\hline \multicolumn{9}{|l|}{ Sex } \\
\hline Male & $180(12.5)$ & 1.3 & {$[1.04,1.63]$} & 0.02 & 106 (14.8) & 0.95 & {$[0.72,1.24]$} & 0.68 \\
\hline \multicolumn{9}{|l|}{ Age (years) } \\
\hline $40-49$ & NA & NA & & & $56(9.6)$ & Ref & & \\
\hline 80-99 & $43(12.2)$ & 1.44 & {$[0.96,2.15]$} & 0.08 & $4(16.0)$ & 1.78 & {$[0.59,5.37]$} & 0.31 \\
\hline \multicolumn{9}{|l|}{ Residence } \\
\hline Major city & $153(12.2)$ & Ref & & & 99 (13.3) & Ref & & \\
\hline Inner regional & $56(8.8)$ & 0.68 & {$[0.49,0.94]$} & 0.02 & 41 (13.2) & 0.97 & {$[0.65,1.44]$} & 0.88 \\
\hline Outer regional & $76(12.2)$ & 0.99 & {$[0.73,1.32]$} & 0.92 & $85(21.0)$ & 1.78 & {$[1.29,2.46]$} & $<0.001$ \\
\hline Remote & $35(9.5)$ & 0.75 & {$[0.51,1.11]$} & 0.15 & $20(11.0)$ & 0.83 & {$[0.49,1.38]$} & 0.47 \\
\hline Very remote & 24 (11.1) & 0.89 & {$[0.56,1.41]$} & 0.62 & 16 (16.7) & 1.3 & {$[0.73,2.34]$} & 0.37 \\
\hline \multicolumn{9}{|l|}{ Education } \\
\hline
\end{tabular}


English

Language other than English

Self-reported diabetes

No

Yes

Time of last eye exam

Less than one year

Between one and two years

Greater than two years

Never

Race

Oceanian

European

Other

\begin{tabular}{|c|c|c|c|c|c|c|c|}
\hline 320 (10.9) & Ref & & & $252(15.1)$ & Ref & & \\
\hline $24(13.7)$ & 1.3 & {$[0.83,2.04]$} & 0.24 & $9(13.4)$ & 0.79 & {$[0.38,1.63]$} & 0.53 \\
\hline 300 (11.2) & Ref & & & 156 (14.3) & Ref & & \\
\hline $44(10.2)$ & 0.84 & {$[0.60,1.17]$} & 0.3 & 105 (16.3) & 0.97 & {$[0.74,1.29]$} & 0.86 \\
\hline 140 (10.4) & Ref & & & 74 (13.9) & Ref & & \\
\hline $110(8.8)$ & 0.86 & {$[0.66,1.13]$} & 0.28 & 94 (14.6) & 1.13 & {$[0.81,1.57]$} & 0.48 \\
\hline 85 (18.8) & 2.06 & {$[1.52,2.77]$} & $<0.001$ & 74 (17.8) & 1.5 & {$[1.05,2.15]$} & 0.03 \\
\hline $9(18.4)$ & 2.26 & {$[1.06,4.85]$} & 0.04 & $19(13.4)$ & 1.28 & {$[0.73,2.24]$} & 0.39 \\
\hline 235 (10.6) & Ref & & & NA & NA & & \\
\hline $80(12.2)$ & 1.13 & {$[0.86,1.49]$} & 0.37 & & & & \\
\hline $29(12.8)$ & 1.28 & {$[0.85,1.94]$} & 0.24 & & & & \\
\hline
\end{tabular}

$29(12.8) \quad 1.28 \quad[0.85,1.94] \quad 0.24$

Odds ratios (OR) of severe refractive error estimated via logistic regression, adjusting for age and sex

Table 4: Estimated association between severe uncorrected refractive error and co-existing ocular conditions in the National Eye Health Survey.

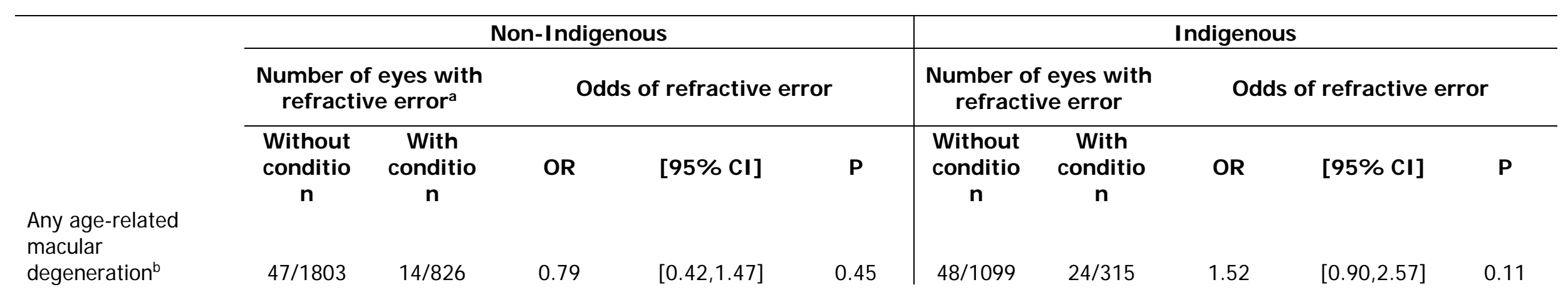

This article is protected by copyright. All rights reserved. 
Probable or definite glaucoma 78/2946 0/152 Undefined

Any diabetic

retinopathyc

$40 / 194$

$9 / 265 \quad 181$

Any cataract

49/2978

$29 / 120$

1.81

$[0.86,3.80]$
$[\mathbf{1 4 . 8 2}, \mathbf{4 4 . 5 4}$

25.69

$74 / 2467$

$4 / 631$

0.21

[0.07,0.60]

\begin{tabular}{c|cc}
- & $91 / 1686$ & $0 / 52$ \\
0.12 & $48 / 990$ & $19 / 331$ \\
$<\mathbf{0 . 0 0 1}$ & $57 / 1634$ & $34 / 104$ \\
& & \\
$\mathbf{0 . 0 0 4}$ & $85 / 1596$ & $6 / 142$
\end{tabular}

Undefined

Cataract surgery

a) Number of participants with refractive error / number participants gradable

b) 793 eyes not able to be graded for age-related macular degeneration

c) 1,304 eyes not able to be graded for diabetic retinopathy

$\mathrm{Cl}=$ confidence interval; OR = odds ratio from age and sex adjusted logistic regression. 


\section{DISCUSSION}

This paper presents the prevalence and characteristics of severe uncorrected refractive error in a national sample of non-Indigenous and Indigenous Australian adults. Severe uncorrected refractive error was common, with $11 \%$ of nonIndigenous Australians aged 50 years and older and 14.5\% of Indigenous Australians aged 40 years and older improving by 2 or more lines on the logMAR chart after auto refraction in one or both eyes. Importantly, over $60 \%$ of all cases did not own glasses or contact lenses for distance viewing (i.e. they were uncorrected rather than sub-optimally corrected) and $80 \%$ had a spherical equivalent auto refraction ranging between $-2.00 \mathrm{D}$ and $+2.00 \mathrm{D}$.

At first glance, the prevalence of uncorrected refractive error amongst nonIndigenous Australians in the NEHS is similar to that of previous Australian reports (BMES ${ }^{11}=10 \%$; VIP ${ }^{10}=10.2 \%$ ). However, comparisons are difficult as the BMES 11 ( $\geq 2$ lines improvement in those with PVA $\leq 6 / 9$ ) and the VIP 10 ( $\geq 1$-line improvement in those with PVA $\leq 6 / 6-2$ ) adopted more conservative definitions for uncorrected refractive error. Given that (1) there is currently no universal definition for severe uncorrected refractive error; (2) $6 / 12$ is a legal requirement for driving in Australia; and (3) $<6 / 12$ is a commonly used definition for visual impairment within epidemiological research in Australia ${ }^{13,22}$ and Internationally, 23,24 we chose a more severe definition (PVA $<6 / 12$ accompanied by improvement of $\geq 2$ lines) that represents visually significant cases that should be prioritised for targeted resource allocation. Furthermore, our definition was inclusive of unilateral severe uncorrected refractive error given the known impact of unilateral vision loss on several visual domains, including stereopsis, visual-motor coordination, depth perception and spatial orientation. ${ }^{25,26}$ In addition, evidence suggests that people with unilateral vision loss are more prone to motor vehicle crashes, ${ }^{27}$ have a greater propensity for falling, have poorer mental and physical health than the general population ${ }^{28}$ and 
are more likely to have an unskilled manual labour job. ${ }^{29}$ Despite this, we are able to recalculate the prevalence according to a more severe secondary definition reported in the same study by the BMES. ${ }^{11}$ This more severe definition of uncorrected refractive error included an improvement of 15 or more letters (3 lines on the LogMAR chart) with a PVA of $<6 / 12 .{ }^{11}$ After adjustments, we noted a lower prevalence of bilateral severe uncorrected refractive error than the BMES in nonIndigenous participants aged 50 years and over (BMES $=4.6 \%$ vs. NEHS $=1.7 \%$ ). The lower prevalence reported in the NEHS may be partly attributed to an improvement in utilisation rates of optometric services amongst non-Indigenous Australians over the past two decades. ${ }^{30}$

Over the past decade, several important and effective initiatives have been implemented within Indigenous populations to provide free or subsidised spectacles for refractive errors. ${ }^{31}$ For Indigenous Australians aged 40 years and over in the NEHS, the prevalence of bilateral severe uncorrected refractive error was $5.2 \%$, more than double that of the non-Indigenous population (2.5\%). This finding, coupled with the fact that $65 \%$ of Indigenous participants with severe uncorrected refractive error did not own glasses or contact lenses for distance viewing, highlights that continued improvements are required to provide equitable access to spectacle correction in Indigenous communities. Our data identified that older Indigenous Australians and those residing in outer regional locations may benefit the most from focussed resource allocation. There is real-world evidence that spherical equivalent correction (only) derived from an autorefractor can be effective for correcting patients with low degrees of astigmatism. ${ }^{32}$ While we acknowledge this is not optimal, given close to $70 \%$ of Indigenous participants with severe uncorrected refractive error had a cylinder equal to or less than 1 dioptre, there may be a case for spherical lenses that fit frames with standardised apertures to be stocked in Aboriginal Health Services in the most inaccessible regions of Australia and health workers could dispense spectacles using autorefraction results. 
There was no significant difference between the mean cylindrical power and spherical equivalent auto refraction of Indigenous and non-Indigenous participants with severe uncorrected refractive error in the NEHS. For both Indigenous and nonIndigenous populations, low hyperopia ( $+2.00 \mathrm{D}$ or less) accounted for a large proportion of uncorrected cases (non-Indigenous $=50 \%$; Indigenous $=43 \%$ ). This finding is not surprising given a hyperopic refractive shift is common with advancing age due to a reduction in the influences of the ciliary muscle and/or zonular fibers causing positional and curvature changes of the natural lens. ${ }^{33,34}$ Low degrees of myopia $(-2.00 \mathrm{D}$ or less) also contributed significantly (non-Indigenous $=32 \%$; Indigenous $=34 \%$ ) to uncorrected refractive error cases in the NEHS. Given that the presence of cataract was associated with a 25.7-fold and 12.3-fold increased likelihood of uncorrected refractive error in non-Indigenous and Indigenous Australians, respectively, it is likely that a myopic shift as a result of increasing nuclear lens opacity is responsible for many cases. In both of these instances (agerelated cataract and age-related hyperopia), participants are not likely to have relied on distance glasses earlier in life, as supported by our finding that over $60 \%$ of severe uncorrected refractive error cases did not own glasses for distance viewing. As a result, it appears that the high uncorrected rates in the present study are attributable to the fact that most new cases of severe refractive error in this population are slow progressing, and therefore gradual changes in VA may go unnoticed.

Approximately $35 \%$ of non-Indigenous and Indigenous participants with severe uncorrected refractive error self-reported using distance correction. This means they either had under-corrected refractive error or they did not present with their corrective lenses at the time of examination. It is possible that the inclusion of participants in the latter category may have overestimated the prevalence of severe uncorrected refractive error in the present study. Regardless, the authors feel that the inclusion of these participants within the definition of presenting severe 
uncorrected refractive error best reflects current visual function and glasses utilisation amongst the Australian non-Indigenous and Indigenous adult population. Previous literature has reported an age-related hyperopic shift in refraction typically in persons aged up to 70 years, followed by a myopic shift in refraction thereafter. ${ }^{35}$ In light of this, it may be speculated that a failure to observe an association between age and severe uncorrected refractive error in the 80-99 year age group in both Indigenous and non-Indigenous participants could be a result of the contribution of nuclear cataract causing a mild myopic shift that neutralizes the preceding agerelated hyperopic shift in this age group. ${ }^{36}$ In addition, this finding may also be partly attributable to the known association between age and cataract surgery coverage in the NEHS, ${ }^{37}$ where participants aged 80-99 years are more likely to be pseudophakic and have a post-operative PVA that is better than $6 / 12$.

The strengths of this study include the multi-stage cluster sampling design and the inclusion of a large sample of non-Indigenous and Indigenous Australians. A number of limitations must also be considered. First, we did not collect data on the current driving status of participants and therefore we were unable to ascertain the proportion of severe uncorrected refractive error cases that were still driving and whether stricter requirements need to be implemented by relevant licensing departments. Second, data on income level may have been useful to further elucidate the association between socioeconomic status and glasses wear and/or likelihood of updating a prescription. Third, we did not perform subjective refraction, but rather relied on the refraction from an autorefractor. Nonetheless, there is evidence that refractions measured by autorefractors can correct vision close to that of subjective refractions. ${ }^{32,38,39}$ Furthermore, the accuracy of autorefractors have been shown to be sufficient for screening purposes. ${ }^{40}$ Lastly, auto-refraction measurements were only available amongst those participants with PVA $<6 / 12$ in one or both eyes. As such, we were unable to conduct prevalence estimates using the more conservative visual acuity thresholds that were adopted in the BMES ( $\geq 2$ 
lines improvement in those with PVA $\leq 6 / 9$ ) and Melb VIP ( $\geq 1$-line improvement in those with PVA $\leq 6 / 6-2)$. However, insights into resource allocation for those with severe uncorrected refractive error can still be taken away from the results of the current study.

The results of this study demonstrate that severe uncorrected refractive error is common among the older non-Indigenous and Indigenous Australians. Nearly all cases fell within the low hyperopic and myopic range, most did not own glasses for distance viewing $(60 \%)$, and those who had not had an eye examination in the previous 2 years were at higher odds of severe uncorrected refractive error. These findings provide evidence that increased public awareness of the importance of regular optometric examinations are required, particularly amongst high risk groups including non-Indigenous Australians residing in urban areas and older Indigenous Australians residing in Outer Regional geographical areas.

\section{Acknowledgements}

In-kind support was received from our industry and sector partners, OPSM, Carl Zeiss, Designs for Vision, the Royal Flying Doctor Service, Optometry Australia and the Brien Holden Vision Institute. We would like to specifically acknowledge OPSM, who kindly donated sunglasses valued at $\$ 130$ for each study participant.

The Centre for Eye Research Australia (CERA) and Vision 2020 Australia wish to recognise the contributions of all the NEHS project steering committee members (Professor Hugh Taylor, Dr Peter van Wijngaarden, Jennifer Gersbeck, Dr Jason Agostino, Anna Morse, Sharon Bentley, Robyn Weinberg, Christine Black, Genevieve Quilty, Louis Young and Rhonda Stilling) and the core CERA research team who assisted with the survey field work (Joshua Foreman, Pei Ying Lee, Rosamond Gilden, Larissa Andersen, Benny Phanthakesone, Celestina Pham, Alison Schokman, Megan Jackson, Hiba Wehbe, John Komser and Cayley Bush). Furthermore, we would like to acknowledge the overwhelming support from all collaborating 
Indigenous organisations who assisted with the implementation of the survey, and the Indigenous health workers and volunteers in each survey site who contributed to the field work.

This article is protected by copyright. All rights reserved. 


\section{REFERENCES}

1. Attebo K, Mitchell P, Smith W. Visual acuity and the causes of visual loss in Australia. The Blue Mountains Eye Study. Ophthalmology 1996; 103(3): 357-64.

2. VanNewkirk MR, Weih L, McCarty CA, Taylor HR. Cause-specific prevalence of bilateral visual impairment in Victoria, Australia: the Visual I mpairment Project. Ophthalmology 2001; 108(5): 960-7.

3. Kat J, Tielsch J M, Sommer A. Prevalence and risk factors for refractive errors in an adult inner city population. Investigative ophthalmology \& visual science 1997; 38(2): 334-40.

4. Klein R, Klein BE, Linton KL, De Mets DL. The Beaver Dam Eye Study: visual acuity. Ophthalmology 1991; 98(8): 1310-5.

5. Lewallen S, Lowdon R, Courtright P, Mehl GL. A population-based survey of the prevalence of refractive error in Malawi. Ophthalmic Epidemiol 1995; 2(3): 1459.

6. Wolfram C, Höhn R, Kottler U, et al. Prevalence of refractive errors in the European adult population: the Gutenberg Health Study (GHS). 2014; 98(7): 85761.

7. Sherwin J C, Lewallen S, Courtright PJ BJ oO. Blindness and visual impairment due to uncorrected refractive error in sub-Saharan Africa: review of recent population-based studies. 2012; 96(7): 927-30.

8. Coleman AL, Yu F, Keeler E, Mangione CM. Treatment of uncorrected refractive error improves vision-specific quality of life. Journal of the American Geriatrics Society 2006; 54(6): 883-90.

9. Owsley C, McGwin G, Jr., Scilley K, Meek GC, Seker D, Dyer A. Effect of refractive error correction on health-related quality of life and depression in older nursing home residents. Archives of ophthalmology (Chicago, //I : 1960) 2007; 125(11): 1471-7. 
10. Liou HL, McCarty CA, J in CL, Taylor HR. Prevalence and predictors of undercorrected refractive errors in the Victorian population. American journal of ophthalmology 1999; 127(5): 590-6.

11. Thiagalingam S, Cumming RG, Mitchell P. Factors associated with undercorrected refractive errors in an older population: the Blue Mountains Eye Study. The British journal of ophthalmology 2002; 86(9): 1041-5.

12. Landers J, Henderson T, Craig J. The prevalence and causes of visual impairment in indigenous Australians within central Australia: the Central Australian Ocular Health Study. The British journal of ophthalmology 2010; 94(9): 1140-4. 13. Taylor HR, Xie J, Fox S, Dunn RA, Arnold AL, Keeffe JE. The prevalence and causes of vision loss in Indigenous Australians: the National Indigenous Eye Health Survey. The Medical journal of Australia 2010; 192(6): 312-8.

14. Foreman J, Keel S, van Wijngaarden $\mathrm{P}$, et al. Prevalence and Causes of Visual Loss Among the Indigenous Peoples of the World: A Systematic Review. JAMA ophthalmology 2018.

15. AlHW. Vision problems among older Australians. 2005 (accessed 10th of February 2017).

16. Foreman J, Keel S, Dunn R, van Wijngaarden P, Taylor HR, Dirani M.

Sampling methodology and site selection in the National Eye Health Survey (NEHS): an Australian population-based prevalence study. Clinical \& experimental ophthalmology 2016.

17. Foreman J, Keel S, van Wijngaarden P, Taylor HR, Dirani M. Recruitment and Testing Protocol in the National Eye Health Survey: A Population-Based Eye Study in Australia. Ophthalmic Epidemio/2017: 1-11.

18. Ferraro J G, Pollard T, Muller A, Lamoureux EL, Taylor HR. Detecting cataract causing visual impairment using a nonmydriatic fundus camera. American journal of ophthalmology 2005; 139(4): 725-6. 
19. Keel S, Xie J, Foreman J, van Wijngaarden P, Taylor HR, Dirani M. The Prevalence of Diabetic Retinopathy in Australian Adults with Self-Reported Diabetes: The National Eye Health Survey. Ophthalmology 2017; 124(7): 977-84.

20. Ferris FL, 3rd, Wilkinson CP, Bird A, et al. Clinical classification of age-related macular degeneration. Ophthalmology 2013; 120(4): 844-51.

21. Keel S, Xie J, Foreman J, et al. Prevalence of glaucoma in the Australian National Eye Health Survey. The British journal of ophthalmology 2018.

22. Taylor HR, Keeffe J E, Vu HT, et al. Vision loss in Australia. The Medical journal of Australia 2005; 182(11): 565-8.

23. Lamoureux EL, Chong E, Wang JJ, et al. Visual impairment, causes of vision loss, and falls: the singapore malay eye study. Investigative ophthalmology \& visual science 2008; 49(2): 528-33.

24. Varma R, Ying-Lai M, Klein R, Azen SP. Prevalence and risk indicators of visual impairment and blindness in Latinos: the Los Angeles Latino Eye Study.

Ophthalmology 2004; 111(6): 1132-40.

25. Fielder AR, Moseley MJ. Does stereopsis matter in humans? Eye (London, England) 1996; 10 ( Pt 2): 233-8.

26. O'Connor AR, Birch EE, Anderson S, Draper HJIo, science v. The functional significance of stereopsis. 2010; 51(4): 2019-23.

27. Keeney K, Garvey J. The dilemma of the monocular driver and Keeney AmJ Ophthal 1981; 91(6): 801-3.

28. Vu HT, Keeffe JE, McCarty CA, Taylor HR. Impact of unilateral and bilateral vision loss on quality of life. The British journal of ophthalmology 2005; 89(3): 3603.

29. Rahi JS, Cumberland PM, Peckham CSJ O. Visual impairment and visionrelated quality of life in working-age adults: findings in the 1958 British birth cohort. 2009; 116(2): 270-4. 
30. Foreman J, Xie J, Keel S, Taylor HR, Dirani M. Utilization of eye health-care services in Australia: the National Eye Health Survey. Clinical \& experimental ophthalmology 2017.

31. Anjou MD, Boudville Al, Taylor HR. Correcting Indigenous Australians' refractive error and presbyopia. Clinical \& experimental ophthalmology 2013; 41(4): 320-8.

32. Treacy MP, Treacy MG, Dimitrov BD, Seager FE, Stamp MA, Murphy CC. A method for the prescription of inexpensive spectacles by non-specialist healthcare workers: S-Glasses. Eye (London, England) 2013; 27(4): 474-9.

33. Attebo K, Ivers RQ, Mitchell P. Refractive errors in an older population: the Blue Mountains Eye Study. Ophthalmology 1999; 106(6): 1066-72.

34. Wang Q, Klein BE, Klein R, Moss SE. Refractive status in the Beaver Dam Eye Study. Investigative ophthalmology \& visual science 1994; 35(13): 4344-7.

35. Kempen J H, Mitchell $P$, Lee $K E$, et al. The prevalence of refractive errors among adults in the United States, Western Europe, and Australia. Archives of ophthalmology (Chicago, III : 1960) 2004; 122(4): 495-505.

36. Samarawickrama C, Wang J J, Burlutsky G, Tan AG, Mitchell P. Nuclear cataract and myopic shift in refraction. American journal of ophthalmology 2007; 144(3): 457-9.

37. Foreman J, Xie J, Keel S, et al. Cataract surgery coverage rates for Indigenous and non-Indigenous Australians: the National Eye Health Survey. The Medical journal of Australia 2017; 207(6): 256-61.

38. Cleary G, Spalton DJ, Patel PM, Lin PF, Marshall J. Diagnostic accuracy and variability of autorefraction by the Tracey Visual Function Analyzer and the ShinNippon NVision-K 5001 in relation to subjective refraction. Ophthalmic \& physiological optics : the journal of the British College of Ophthalmic Opticians (Optometrists) 2009; 29(2): 173-81. 
39. Sun J K, Aiello LP, Cavallerano J D, et al. Visual acuity testing using autorefraction or pinhole occluder compared with a manual protocol refraction in individuals with diabetes. Ophthalmology 2011; 118(3): 537-42.

40. Tuncer I, Zengin MO, Karahan E. Comparison of the Retinomax hand-held autorefractor versus table-top autorefractor and retinoscopy. International journal of ophthalmology 2014; 7(3): 491-5. 


\section{University Library}

\section{- M M N E R VA A gateway to Melbourne's research publications}

Minerva Access is the Institutional Repository of The University of Melbourne

Author/s:

Keel, S;McGuinness, MB;Foreman, J;Scheetz, J;Taylor, HR;Dirani, M

Title:

Prevalence, associations and characteristics of severe uncorrected refractive error in the Australian National Eye Health Survey

Date:

2019-10-16

Citation:

Keel, S., McGuinness, M. B., Foreman, J., Scheetz, J., Taylor, H. R. \& Dirani, M. (2019). Prevalence, associations and characteristics of severe uncorrected refractive error in the Australian National Eye Health Survey. CLINICAL AND EXPERIMENTAL OPHTHALMOLOGY, 48 (1), pp.14-23. https://doi.org/10.1111/ceo.13647.

Persistent Link:

http://hdl.handle.net/11343/286509 